\title{
COLLINEATION AS A MODE OF MOTION.*
}

\author{
BY MAXIME BÔCHER, PH.D.
}

Is the following paper I have attempted to give an account of some very simple matters, which, although familiar to many, appear to have attracted but little attention in this country. The subject, however, has never, as far as I know, been presented from precisely the point of view here adopted.

Perhaps the most important difference between the old and the new geometry lies in the extended use made during the present century of geometric transformations. $\dagger$ The change which has come about in this direction is due in part to the influence of certain branches of applied mathematics in which one has to deal not merely with geometric configurations but also with certain changes which these configurations are forced to undergo. There are however two distinct ways of looking at a transformation. First we may consider the original and the transformed figure as standing side by side, or even as occupying portions of the same space, the latter being in a certain sense a picture of the former; or secondly, we may consider the original figure to be gradually deformed according to a given law into the transformed figure. Each of these points of view can be traced to a physical origin. Perspective and allied subjects strikingly illustrate the first, while the second will most naturally be adopted in hydrodynamics, the theory of elasticity, etc. Now while the first of the above mentioned ways of looking at a transformation has the advantage of introducing no unnecessary element into the consideration, the second in turn has the advantage of making the idea of a transformation lose much of its abstractness, for by its aid we are enabled to see the points of the original figure rearrange themselves by a gradual motion into the transformed figure.

I wish to illustrate this way of looking at a transformation as a mode of motion by considering one of the simplest of transformations, the so-called linear transformation or collineation, $\$$ and for the sake of simplicity I will confine myself to two dimensions.

\footnotetext{
* Lecture delivered June 4, 1892, before the New York Mathematical Society.

+ The following remarks should be understood to apply only to point transformations, i.e., to transformations which carry points over into points.

$\ddagger$ The word collineation seems to be by far the best name for this transformation, not only because it is as applicable in synthetic as in analytic geometry, but also because the ambiguity which arises in speaking of a
} 
Using any system of trilinear coordinates $\left(x_{1}, x_{2}, x_{8}\right)$, a collineation will be expressed by the linear formulæ:

$$
\begin{aligned}
& \rho x_{1}{ }^{\prime}=a_{1} x_{1}+a_{2} x_{2}+a_{3} x_{3}, \\
& \rho x_{2}^{\prime}=b_{1} x_{1}+b_{2} x_{2}+b_{3} x_{3}, \\
& \rho x_{3}{ }^{\prime}=c_{1} x_{1}+c_{2} x_{2}+c_{3} x_{3},
\end{aligned}
$$

( $\rho$ being an undetermined factor of proportionality).

It is however well known that in general a collineation leaves three points of the plane fixed while all other points are carried over into new positions. If now these three fixed points be taken as the vertices of the triangle of reference, the collineation will evidently be expressed by the very simple formulæ :

$$
\rho x_{1}^{\prime}=a x_{1}, \quad \rho x_{2}^{\prime}=b x_{2}, \quad \rho x_{3}{ }^{\prime}=c x_{3} .
$$

These formulæ tell us into what position each point of the plane is carried over by the transformation; they give us, however, no clue as to what path it is advisable to regard as traversed by each point in passing from its original to its final position.

To determine this, let us first consider the case in which two of the fixed points are the circular points at infinity, the third (finite) fixed point being denoted by the letter $O$. This collineation may be shown by a simple calculation, to consist of a rotation of the plane as a whole about the point $O$ combined with a uniform stretching of the plane away from (or contraction of the plane towards) this same point. In the case of a rotation, however, each point will naturally be regarded as moving from its, original to its final position along the arc of a circle whose centre is at $O$; in the case of expansion or contraction on the other hand, the lines of motion will be the straight lines through $O$, the motion taking place away from $O$ in the case of expansion and towards $O$ in the case of contraction; the amount of the motion in any case being proportional to the distance from $O$. If, then, we have a combination of rotation and expansion (or contraction) the lines of motion will evidently be equal logarithmic spirals with pole at $O$. Taking $O$ as the origin of a system of polar coordinates, the equation of this family of logarithmic spirals will be :

$$
r=A e^{k \phi},
$$

linear transformation without specifying what system of coordinates we use is a very real objection, as there are other coordinates besides trilinear (for example, Darboux': tetracyclic coordinates) in which linear transformations are actually considered. The term "homographic transformation," introduced by Chasles, is not as expressive as the term collineation used some years before by Mobius. It does not seem as though Chasles' ignorance of the German language could justify us in adopting his poorer names in place of the original better ones. 
where $k$ is a constant determining the shape of the spirals, while $A$ is a parameter varying from one member of the family to another. We shall find it more convenient to write in place of $k$ the quotient $k_{1} / k_{2}$.

Let us now introduce a system of trilinear coordinates in which the vertices of the triangle of reference are the circular points at infinity and the point $O$. We will denote these coordinates by the letters $(\xi, \eta, t)$, the first two referring to the imaginary sides of the triangle of reference through $O$, while the last refers to the line at infinity. In this system of homogeneous "circular" coordinates the equation of the above mentioned family of logarithmic spirals is readily found to be :

$$
\xi^{k_{2}+i k_{1}} \eta^{k_{2}-i k_{1}} t^{-2 k_{2}}=A^{2 k_{2}},
$$

or since $k_{1}, k_{2}, A$ are any constants :

$$
\xi^{a} \eta^{\beta} t^{\gamma}=C
$$

provided that $\alpha+\beta+\gamma=0$.

We can now write out at once the equations of the lines of motion in the general case where we have as fixed points any three points of the plane, for we have merely to project the point $O$ and the circular points at infinity in the special case we have just considered into any other three points in order that the logarithmic spirals should go over into the lines of motion of a general collineation. The equation of the family of lines of motion, referred to the triangle of reference whose vertices are the fixed points, is then :

$$
x_{1}^{\alpha} x_{2}^{\beta} x_{3}^{\gamma}=C \text {, }
$$

where $C$ is the variable parameter of the family while the constants $\alpha, \beta, \gamma$ (which are connected by the relation $\alpha+\beta+\gamma=0$ ) depend upon the coefficients $a, b, c$ of the linear transformation (2) above.*

The fixed points of a collineation may be all real, or one of them may be real and the other two conjugate imaginary. The last of these two possibilities need not detain us long, as it may be obtained by a real projection from the special case considered above where two of the fixed points were the circular points at infinity. In it we shall have in our triangle of reference one vertex and the side opposite real, while the remaining vertices and sides are imaginary. The lines of motion will have a spiral form, each consisting of an infinite

* It is easily found that $\alpha, \beta, \gamma$ are proportional respectively to $\log \frac{c}{b}, \log \frac{a}{c}, \log \frac{b}{a}$. 
number of coils about the real fixed point. As these coils become larger they will become more and more elongated in the direction farthest from the real side of the triangle of reference, until each coil finally assumes a hyperbolic form, running out on the side of the fixed point farthest from the real side of the triangle of reference through infinity, and completing itself on the other side of the real line in question. These hyperbolic coils ultimately approach the real side of the triangle of reference asymptotically.

When all of the fixed points are real, however, the lines of motion will have completely lost their spiral character. Here again there are two cases to consider, according as the three coefficients $a, b, c$ of the transformation have all the same sign, or one of them a different sign from the other two. The three indefinitely extended sides of the triangle of reference divide the plane into four parts, one finite and the other three infinite. We may speak of each of these parts as "triangles," in spite of the fact that each of the three infinite triangles appears to be divided into two distinct portions by the line at infinity. Using this terminology we may say that when all three coefficients $a, b, c$ have the same sign, the interior of each of these four triangles is transformed into itself ; but when one of the three coefficients has a different sign from the other two the triangles are interchanged in pairs. We will begin with the simpler of the two cases, in which each triangle is transformed into itself. The lines of motion in this case will be found to lie as follows :-*

Within the finite triangle the lines of motion all start from the vertex corresponding to the smallest of the three coefficients, $\uparrow$ and run without singularity to the vertex corresponding to the largest of them; at each of their extremities these curves are tangent to the side of the triangle joining that extremity with the vertex corresponding to the coefficient which lies in magnitude between the other two. The side of the triangle joining the vertices which correspond to the greatest and the smallest coefficient is, of course, itself a line of motion, and the same is true of the broken line consisting of the other two sides of the triangle.

* One way of seeing this is to consider first the special case in which the triangle of reference consists of two lines at right angles to one another and the line at infinity, and then to project this into the general case. In the special case just mentioned we have to deal with the same transformation which occurs in the theory of small irrotational strains (see for instance MrNcHIN, Uniplaner Kinematics, chap. v.). It is interesting to notice that this is a case in which the idea of lines of motion is naturally suggested by a physical application.

$\uparrow$ The coefficients $a, b, c$ are said to correspond to the vertices opposite the sides $x_{1}=0, x_{2}=0, x_{3}=0$ respectively. 
The lines of motion within each of the other tbree triangles will be precisely like those just described, the difference in appearance being due to the fact that these triangles themselves extending through infinity, some of the lines of motion in one of these three triangles and all of the lines of motion in the other two will run through infinity on their way from the vertex corresponding to the smallest of the coefficients to the one corresponding to the largest.

It should be noticed that while these curves are in general transcendental and extend only between two fixed points of the collineation where they suddenly stop, we can find special collineations for which the curves are algebraic and all of any degree we please. The family of curves will not look particularly different in these cases from what it does when the curves are transcendental, but the curves themselves will have a different shape. They will now no longer stop at the two fixed points just mentioned, but will continue beyond them into another triangle, having singularities in these points when their degree is higher than the second (in the case of cubics, a cusp in one point, and a point of inflection in the other). The case where the lines of motion are conics all tangent at the extremities of one of the sides of the triangle of reference to the other two sides deserves special mention owing to its frequent occurrence in projective geometry.*

Coming now to the case where one of the coefficients of the transformation has a different sign from the other two, it is readily seen that the lines of motion are here imaginary although each contains an infinite number of real points. Every point of the plane is therefore carried over from its original to its final position through an imaginary path. We are therefore unable to follow the motion of the points of the plane. It is however possible to break up the transformation into two parts, one very simple, the other more complicated but having real lines of motion. Thus for instance we can break up the collineation :

$$
\rho x_{1}^{\prime}=-2 x_{1}, \quad \rho x_{2}^{\prime}=3 x_{2}, \quad \rho x_{3}^{\prime}=5 x_{3},
$$

into the two collineations :

$$
\begin{array}{lll}
\rho \bar{x}_{1}=-x_{1}, & \rho \bar{x}_{2}=x_{2}, & \rho \bar{x}_{3}=x_{3} ; \\
\rho x_{1}^{\prime}=2 \bar{x}_{1}, & \rho x_{2}^{\prime}=3 \bar{x}_{2}, & \rho x_{3}^{\prime}=5 \bar{x}_{3} .
\end{array}
$$

* We may of course have conics as lines of motion when two of the fixed points of the collineation are imaginary, rotation of the plane about a point being a special case of this. In fact, whenever the lines of motion are conics, whether the fixed points are real or imaginary, the collineation will be merely a non-euclidian rotation if we take one of these conics as the absolute. 
The second of these has as its lines of motion the real transcendental curves discussed above, while I may perhaps be allowed to describe the first as a "projective reflection" with regard to the side $x_{1}=0$ and the opposite vertex. The nature of the transformation brought about by this projective reflection is so simple that it need not be discussed here, and that we do not need the assistance of lines of motion to get a perfectly clear idea of it.* It is of course merely the projective generalization of ordinary reflection; reflection with regard to the axis of $X$, for instance, in a system of rectan. gular coordinates, being merely a projective reflection with regard to this line and the infinitely distant point on the axis of $Y$.

It remains to mention some of the literature connected with this subject. 'The transcendental curves, which we have called the lines of motion of the collineation, occur incidentally in a paper by Clebsch and Gordan in the Mathematische Annalen, vol. I. They were however first systematically considered by Klein and Lie in vol. Iv. of the same journal (1871). The reader is referred to this paper for the modification of the lines of motion which occur in the various special cases (when the collineation has two coincident fixed points, etc.). The very brief indications there given can readily be amplified as has been done in this paper for the general case. The reader will also find in this beautiful paper an account of some of the remarkable properties of these curves, which thus gain an interest far above that attaching as yet to most other transcendental curves, owing to the fact that their properties form to some extent a systematic whole, not a mass of facts more or less ingeniously proved. More important still however is the connection of these lines of motion with Lie's now famous theory of differential equations, $\dagger$ some of the very earliest of Lie's investigations in this direction being contained in the paper just mentioned. By the introduction of infinitesimal transformations it is possible to obtain the lines of motion directly without first considering the special case in which the circular points at infinity are two of the fixed points. We thus find the equation of the lines of motion as the solution of a differential equation.

In still another way must Klein's name be connected with

\footnotetext{
* It should however be noticed that a projective reflection (and therefore any ordinary reflection) may be regarded as having real lines of motion, viz, conics. This will be most readily seen if we consider that the projective reflection with regard to the line at infinity and a finite point is equivalent to a rotation through an angle of $180^{\circ}$ about that point.
}

† See Lie's recently published book on this subject edited by Scheffers. 
this subject. A few preliminary remarks are necessary to explain this. The linear transformation of a single straight line into itself may be studied from precisely the same point of view as we adopted above in the case of two dimensions. Three cases would again present themselves : one in which the two fixed points are imaginary, and two in which they are real. In one of these last the transformation cannot be regarded as a real motion, while in the other two it can. Now the extension of our theory which suggests itself to us here depends upon the fact that the complex points of a straight line can be conveniently represented in a plane of which the line is the axis of reals. The linear transformation of the line will then give us a corresponding transformation of the plane which of course should not be confounded with the collineation discussed above. The coefficients here need no longer be real to give us a real transformation. This new transformation of the plane may also be regarded as a mode of motion and has been so treated by Klein in his lectures for a number of years (see an article by Prof. Cole in the Annals of Mathematics for June, 1890, and part II. chap. I. of the recently published Modulfunctionen of Klein-Fricke). The idea cannot fail to suggest itself that the transformation of the plane which we have called collineation should be generalized in a similar way by representing the complex as well as the real points of the plane. I do not know of this subject having been treated; it would of course lead us into four dimensional space.

Harvard Universtty, June, 1892.

\section{NOTES.}

A Regular meeting of the NEW YorK Mathematical SocIETY was held Saturday afternoon, June 4, at half past two o'clock, the president in the chair. The following persons having been duly nominated, and being recommended by the council, were elected to membership : Dr. James Whitbread Lee Glaisher, Trinity College, Cambridge, England; Mr. Ferdinand Shack, New York, N. Y. The following papers were read : "An expression for the total surface of an ellipsoid in terms of $\sigma$-and $p$-functions, including an application to the surface of a prolate spheroid," by Professor J. H. Boyd ; "On collineation as a mode of motion," by Dr. Maxime Bôcher ; "On Peters' formula for probable error," by Professor W. Woolsey Johnson. 